\title{
Studies of the $\eta$ meson decays with WASA
}

\section{A. Kupsc ${ }^{* a b}$, P. Adlarson ${ }^{a}$, M. Berłowski ${ }^{b}$, H. Bhatt ${ }^{c}$ K. Chandwani ${ }^{c}$, D. Coderre ${ }^{d}$, M. Hodana ${ }^{d e}$, M. Janusz ${ }^{d e}$ Ch. Pauly ${ }^{d}$, Ch. F. Redmer ${ }^{d}$, N. Shah ${ }^{c}$, J. Stepaniak ${ }^{b}$, P. Vlasov ${ }^{d}$, A. Winnemöller ${ }^{f}$, J. Złomanczuk ${ }^{a}$ and L. Yurev ${ }^{d}$}

for the CELSIUS/WASA and the WASA-at-COSY Collaborations

${ }^{a}$ Division of Nuclear and Particle Physics, Department of Physics and Astronomy, Uppsala University, Box 516, 75120 Uppsala, Sweden

${ }^{b}$ High Energy Physics Department, The Andrzej Soltan Institute for Nuclear Studies, ul. Hoza 69, 00-681, Warsaw, Poland

${ }^{c}$ Department of Physics, Indian Institute of Technology Bombay, Powai, Mumbai, 400076 Maharashtra, India

${ }^{d}$ Institut für Kernphysik and Jülich Center for Hadron Physics, Forschungszentrum Jülich, 52425 Jülich, Germany

${ }^{e}$ Institute of Physics, Jagiellonian University, ul. Reymonta 4, 30-059 Kraków, Poland

${ }^{f}$ Institut für Kernphysik, Westfälische Wilhelms-Universität Münster, Wilhelm-Klemm-Str. 9 , 48149 Münster, Germany

E-mail: Andrzej.Kupsc@physics.uu.se

Studies of $\eta$ meson decays with the WASA detector are a major goal of the experimental program at COSY-Jülich. In 2008 a sample of $10^{7} \eta$ mesons was collected in $p d \rightarrow{ }^{3} \mathrm{He} \eta$ reaction close to threshold without trigger bias on the decay channels. The status of the analysis of various decay channels is presented.

6th International Workshop on Chiral Dynamics, CD09

July 6-10, 2009

Bern, Switzerland

\footnotetext{
${ }^{*}$ Speaker.
} 


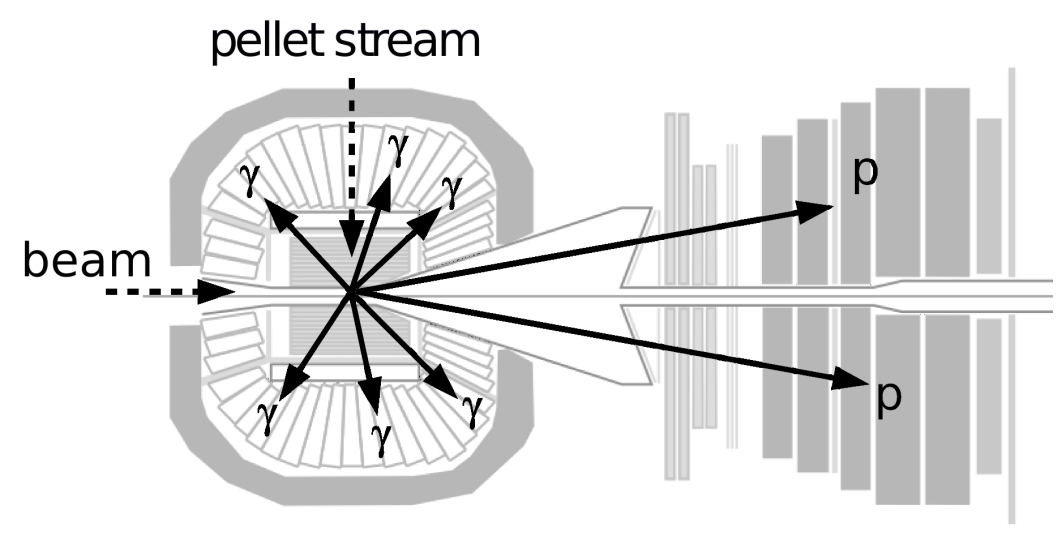

Figure 1: Schematic representation of the WASA detector setup. An example $p p \rightarrow p p 6 \gamma$ event is sketched.

\section{Introduction}

One of the main goals of the experimental programme for the WASA detector at COSY is the study of $\eta$ meson decays [1]. The WASA detector was build at TSL Uppsala and it was used until 2005 at the CELSIUS light ion storage ring [2]. WASA (fig. 1) includes a novel pellet target system allowing for low background and wide angle detection. The design of the detector was optimized for $\pi^{0}, \eta$ decays involving photons and electrons. In addition to the electromagnetic calorimeter the central part includes a superconducting solenoid and a cylindrical mini drift chamber (MDC) with 17 layers of thin-walled $(25 \mu \mathrm{m})$ aluminized mylar tubes. The forward part (FD) consists of layers of plastic scintillators and tracking chambers for measurement of recoil particles. The complete detector system was transported to Forschungszentrum Jülich in 2005, installed at the COSY storage ring and it was operational already after one year. The relocation of the detector had a positive impact for the experimental programme since COSY allows for higher beam energies (above the $p p \rightarrow p p \phi$ threshold) and for investigations of spin observables. In particular studies of the $\eta^{\prime}$ meson decays are now possible. After the transfer the detector was upgraded with a completely new readout system, refurbished scintillator elements and an extension of the forward part for the higher energies.

\section{Status of the analysis of the $\eta$ meson decays}

The initial stage of the $\eta$ meson decay experiments was carried out in $p p \rightarrow p p \eta$ and $p d \rightarrow{ }^{3} \mathrm{He} \eta$ reactions close to threshold at CELSIUS. Results on the Dalitz plot density for the $\eta \rightarrow 3 \pi^{0}$ decay [6] and on the branching ratios of some leptonic decay channels $[4,5]$ were already reported earlier.

During the last two years new data has been collected on $\eta$ decays in $p p$ and $p d$ interactions at COSY. A short production run in April 2007 yielded $10^{6}$ events on $p p \rightarrow p p(\eta \rightarrow 6 \gamma)$ reaction. In this first run, $80 \mathrm{k}$ events were collected in the final $\eta \rightarrow 3 \pi^{0}$ Dalitz plot. The results of this beam time are published in [3].

In the next four week proton-deuteron run period in October $2008 \eta$ decays were studied using the $p d \rightarrow{ }^{3} \mathrm{He} \eta$ reaction at a beam energy of $1 \mathrm{GeV}$. An unbiased data sample of $1.1 \times 10^{7} \eta$ meson decays was collected. Recently in October 2009 an eight week continuation of this experiment was 

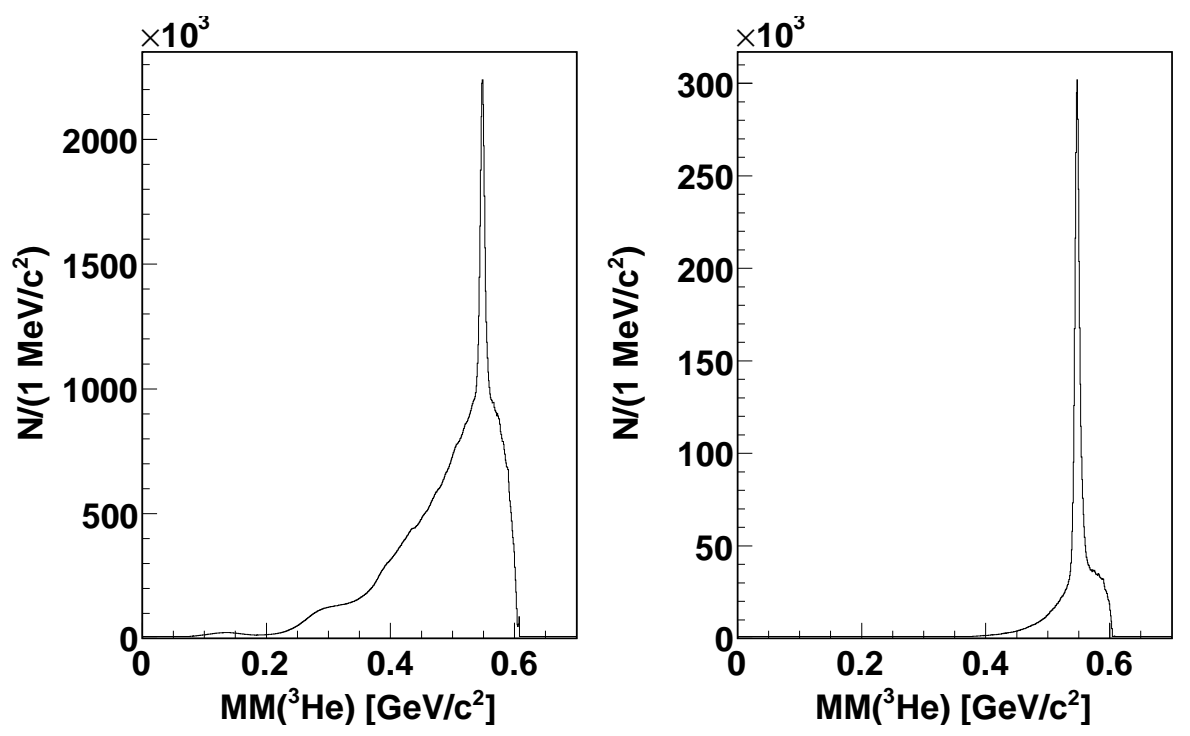

Figure 2: Distribution of ${ }^{3} \mathrm{He}$ missing mass for $p d \rightarrow{ }^{3} \mathrm{He} X$ reaction at $1.0 \mathrm{GeV}$. The trigger was based entirely on ${ }^{3} \mathrm{He}$ signal in FD without bias on the decay system. The plot shows all data collected in 2008 run period. (left) data analysis bases only on the forward detector track - there is about $11 \times 10^{6}$ events in the peak at the $\eta$ meson mass (right) in addition invariant mass of two photons $\geq 300 \mathrm{MeV} / \mathrm{c}^{2}$.

finished and the data sample was increased to $3 \times 10^{7} \eta$ meson decays. In addition few shorter runs aiming for optimization of $\eta$ meson production $p p$ interactions were also carried out.

\section{1 $p p \rightarrow p p \eta$ and $p d \rightarrow{ }^{3}$ He $\eta$ experiments}

The cross section of $p d \rightarrow{ }^{3} \mathrm{He} \eta$ reaction raises quickly and reaches a plateau value of $400 \mathrm{nb}$ already at $2 \mathrm{MeV}$ above the threshold. The requirement of a ${ }^{3} \mathrm{He}$ ion in the final state selects only a very tiny fraction of the total $p d$ cross section (about $0.1 \%$ ). Identification of ${ }^{3} \mathrm{He}$ with respect to protons and deuterons is quite reliable and can be easily implemented on the trigger level. Doing this one obtains trigger rates at maximum luminosity well within the DAQ capabilities without imposing any additional condition on the $\eta$ decay system. Maximum advantage of the $p d \rightarrow{ }^{3} \mathrm{He} \eta$ reaction can be exploited only when running very close to the threshold and tagging and triggering is done using a zero degree spectrometer. This approach was used by the CELSIUS/WASA collaboration for studying leptonic decays [4, 5].

Unfortunately the installation of a zero degree spectrometer is not possible for WASA's new location at COSY. Here the $p d \rightarrow{ }^{3} \mathrm{He} \eta$ reaction is used at slightly higher energy where the ${ }^{3} \mathrm{He}$ ions can be measured in the forward detector which covers scattering angles from $3^{\circ}$ to $18^{\circ}$. Figure 2 shows the missing mass of the reconstructed ${ }^{3} \mathrm{He}$ at $1.0 \mathrm{GeV}$ beam energy. The data were obtained with a trigger requiring ${ }^{3} \mathrm{He}$ in FD and no particular decay channel was selected. In the left panel all data collected in the $2008 p d$ run period are shown. In the right panel after event selection for $\eta \rightarrow \gamma \gamma$ decay.

Due to WASA's calorimeter and MDC particle flux limitations the maximal rate of $\eta$ mesons from the $p d \rightarrow{ }^{3} \mathrm{He} \eta$ reaction is about $10 \eta$ events/s. Therefore the reaction can only be used for studies of not-so-rare $\eta$ decays. Further progress towards rare $\eta$ decays can only be made by 

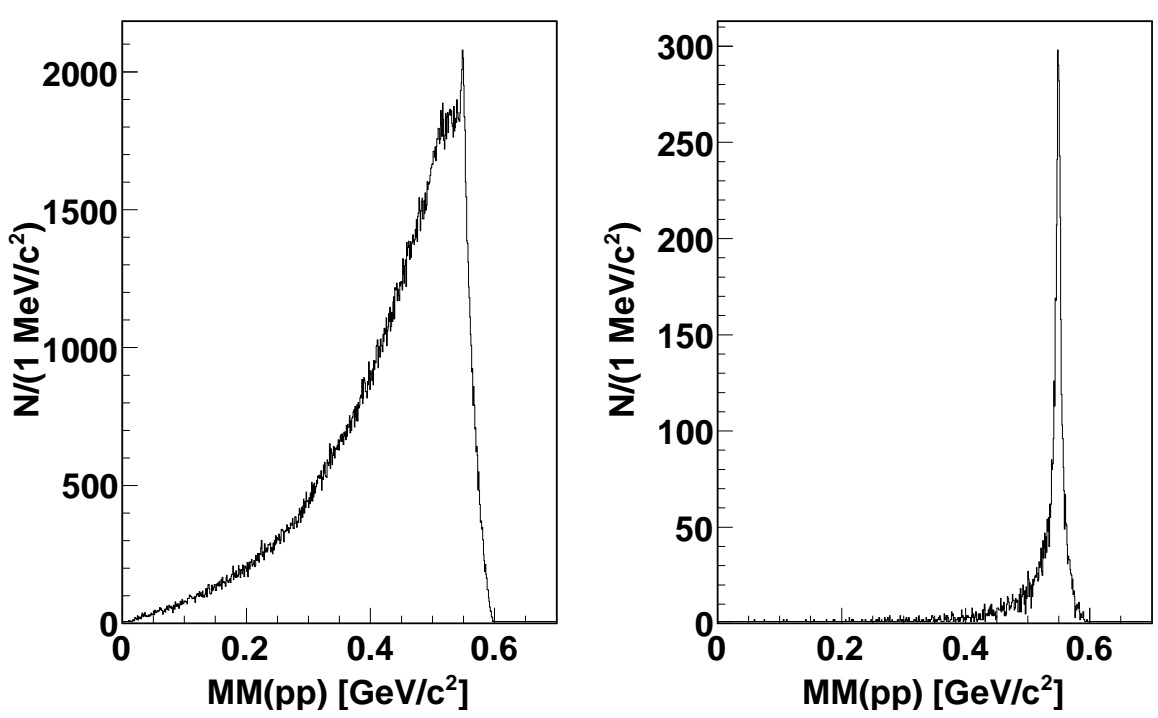

Figure 3: Proton-proton missing mass distributions in $p p \rightarrow p p X$ reaction at $1.4 \mathrm{GeV}$ with trigger requiring two tracks from charged particles in FD and at least two clusters from neutral particles in the calorimeter. (left) data analysis bases only on the forward detector tracks (right) in addition invariant mass of the two photons $\geq 300 \mathrm{MeV} / \mathrm{c}^{2}$.

focusing on the $p p \rightarrow p p \eta$ production reaction. The reaction has 10-20 times larger cross section $(10 \mu \mathrm{b}$ at $1.4 \mathrm{GeV})$ and the inclusive $p p$ cross section is two times lower than for $p d$ interactions. Therefore the $\eta$ meson yield will be higher for a given luminosity and in addition one can use up to two times higher luminosity. The disadvantage of the $p p \rightarrow p p \eta$ reaction is much more difficult tagging. This is illustrated in figure 3 showing missing mass distributions for two reconstructed protons in the FD in a run at $1.4 \mathrm{GeV}$. The trigger condition in this case included requirement of at least two tracks in FD and at least two clusters from neutral particles in the calorimeter. The missing mass plot on the left side was obtained based on all events selected by the trigger, where the right figure shows the $p p$ missing mass after selection of the $\eta \rightarrow \gamma \gamma$ decay channel in the analysis. Dedicated trigger conditions for different $\eta$ decay modes are needed. Due to limited trigger selectivity a production rate of about $100 \eta$ events/s was so far achieved. Preparations of an improved trigger system now are underway.

\section{$2.2 \eta \rightarrow \pi^{0} \pi^{0} \pi^{0}$}

Measurement of Dalitz plot densities of the $\eta \rightarrow \pi^{0} \pi^{0} \pi^{0}$ and $\eta \rightarrow \pi^{+} \pi^{-} \pi^{0}$ decays as well as the $\Gamma\left(\eta \rightarrow \pi^{0} \pi^{0} \pi^{0}\right) / \Gamma\left(\eta \rightarrow \pi^{+} \pi^{-} \pi^{0}\right)$ ratio comprises a very important test of Chiral Perturbation Theory (ChPT).

The decay amplitude of $\eta$ into three pions can be described using the following two Dalitz variables:

$$
x \equiv \frac{1}{\sqrt{3}} \frac{T_{1}-T_{2}}{\langle T\rangle} ; y \equiv \frac{T_{3}}{\langle T\rangle}-1 .
$$

Here $T_{i}$ are the kinetic energies of the pions in the rest frame of the $\eta$ meson, $3\langle T\rangle \equiv T_{1}+T_{2}+T_{3}=$ $m_{\eta}-3 m_{\pi}$ where $m_{\pi}$ is the pion mass and the $\pi^{0}, \pi^{+}$mass difference is neglected. 


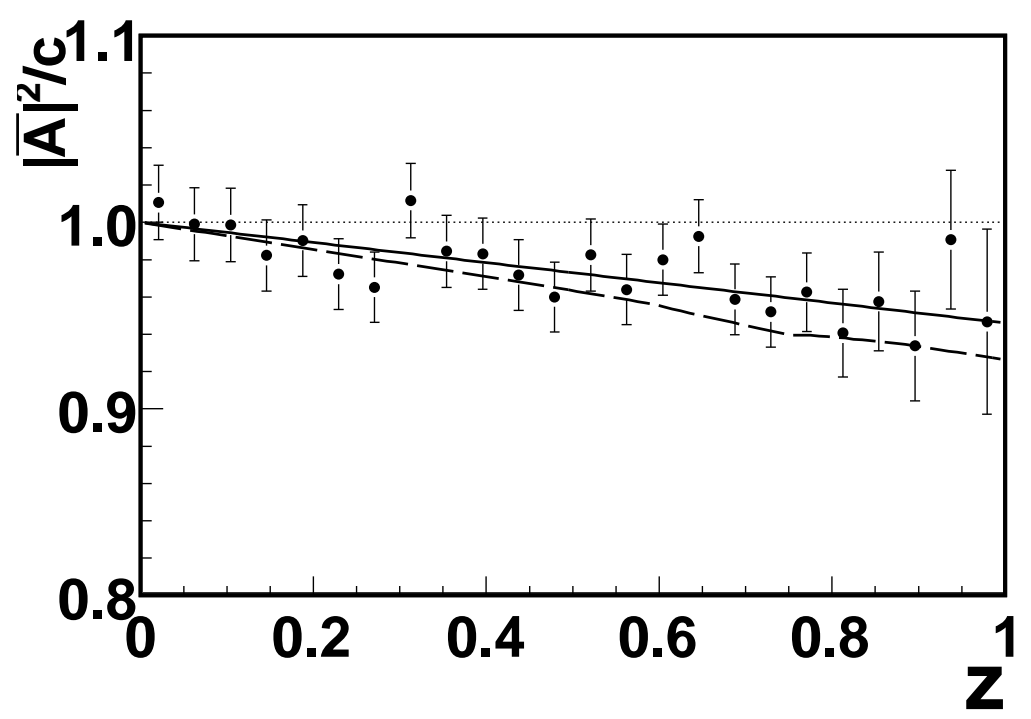

Figure 4: WASA-at-COSY result on $|\overline{\mathscr{A}}|^{2}$ dependence on the $z$ variable for $\eta \rightarrow \pi^{0} \pi^{0} \pi^{0}$ decay [3]. The solid line is a $c(1+2 \alpha z$ ) fit leading to $\alpha=-0.027 \pm 0.008$ (stat) \pm 0.005 (syst). The dashed line is a prediction of the cusp effect [18].

The $\eta \rightarrow \pi^{0} \pi^{0} \pi^{0}$ decay amplitude $(\overline{\mathscr{A}})$ is constant in the leading order of ChPT. Experimentally, a small deviation from a uniform Dalitz Plot density distribution is observed. The lowest term in the expansion about the center of the Dalitz plot is given by:

$$
|\overline{\mathscr{A}}(z)|^{2}=c(1+2 \alpha z)
$$

where $z=x^{2}+y^{2}$ and $c, \alpha$ are the parameters.

The slope $\alpha$ was measured to be negative and small, which leads to a decrease of the Dalitz plot density at the border by a few percent. The explanation of this effect poses a challenge for ChPT which in next-to-leading order predicts a small positive value for $\alpha$ [7]. The large uncertainty of the recently carried out next-to-next-to-leading order (NNLO) calculations [9] does not allow to decide the sign of the slope.

The primary motivation for $\eta \rightarrow \pi^{0} \pi^{0} \pi^{0}$ measurements at WASA was a three sigma discrepancy between Crystal Ball at AGS [13] result from 2001 and preliminary KLOE analysis from 2005 [14]. The discrepancy is now resolved and the reanalyzed KLOE data [15] are consistent with Crystal Ball. The slope $\alpha$ was also measured both by the CELSIUS/WASA [6] and the WASAat-COSY collaborations (figure 4) [3]. The Crystal Ball collaboration has published new results from the MAMI facility $[17,16]$. The results from all experiments are consistent and the weighted average slope value is $\alpha=-0.0312 \pm 0.0017$.

The investigations of the decay has entered a new interesting phase. As a consequence of the pion mass difference, $m_{\pi^{0}}<m_{\pi^{ \pm}}$, the threshold for the $\pi^{0} \pi^{0} \rightarrow \pi^{+} \pi^{-}$process is covered by the $\eta \rightarrow \pi^{0} \pi^{0} \pi^{0}$ phase space. A similar effect as the recently observed cusp in the $K^{+} \rightarrow \pi^{+} \pi^{0} \pi^{0}$ decay by the NA48/2 collaboration [19] is expected. However it will have only minor influence for the shape of the $z$ distribution as shown in figure 4 . 

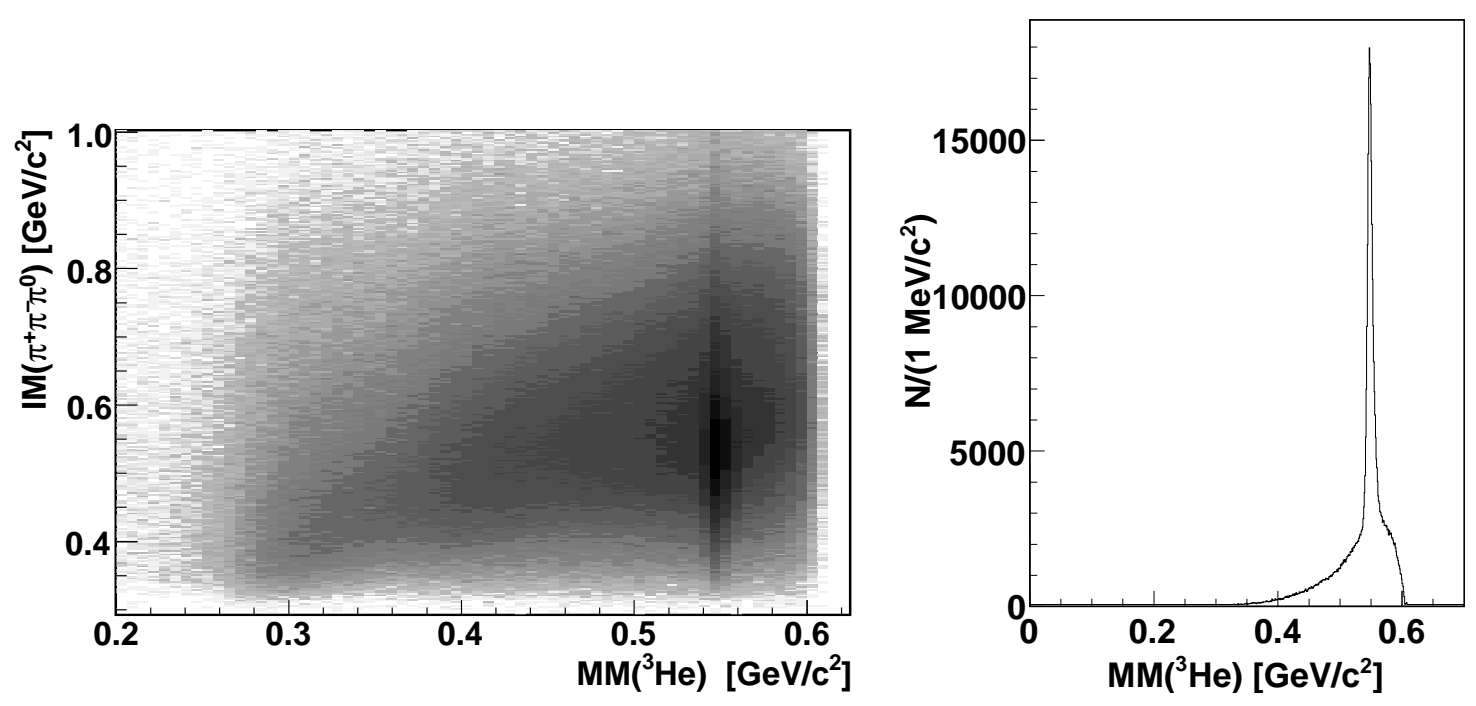

Figure 5: Invariant mass $\left(\pi^{+} \pi^{-} \pi^{0}\right)$ and missing mass $\left({ }^{3} \mathrm{He}\right)$ for an event selection of the $\eta$ decay into $\pi^{+} \pi^{-} \pi^{0}$.

The data for $\eta \rightarrow \pi^{0} \pi^{0} \pi^{0}$ decay are being collected in WASA runs both in $p p$ and $p d$ experiments. For example during two weeks $p p$ run period in November 2008 a data sample corresponding to about $3 \times 10^{5}$ events in the final Dalitz plot was collected. This has to be compared to the $80 \mathrm{k}$ events obtained during the first production run in April 2007 [3].

$2.3 \eta \rightarrow \pi^{+} \pi^{-} \pi^{0}$

For the $\eta \rightarrow \pi^{+} \pi^{-} \pi^{0}$ decay channel Dalitz plot density will depend on both $x$ and $y$ variables. There is one recent precise result from KLOE with $1.3 \cdot 10^{6}$ events [20] in the Dalitz plot. This is an improvement of more than two orders of magnitude in the event statistics compared to all previous measurements. The KLOE results for the Dalitz plot density cannot be reproduced by any of the ChPT calculations.

During the $p d$ run in 2008 WASA has collected a $\eta \rightarrow \pi^{+} \pi^{-} \pi^{0}$ data sample which should lead to about $2 \times 10^{5}$ events in the final Dalitz plot. Together with the data collected in 2009 this will allow us to cross-check the KLOE result. Figure 5 shows the signature of this channel, based on the analysis of the 2008 data.

\section{$2.4 \eta \rightarrow \pi^{+} \pi^{-} \gamma$}

The decay channel $\eta \rightarrow \pi^{+} \pi^{-} \gamma$ allows one to study the box anomaly in QCD. The goal is to extract shape of the photon energy spectrum which should allow to differentiate between the simplest gauge invariant matrix element and the ChPT calculations. Missing mass for the identified $\eta \rightarrow \pi^{+} \pi^{-} \gamma$ channel from WASA-at-COSY data is shown in figure 6 . A first step before constructing the photon energy distribution is a consistency check of the data by inspecting the second kinematic variable in the decay: the distribution of the $\pi^{+}$direction in the di-pion center of 

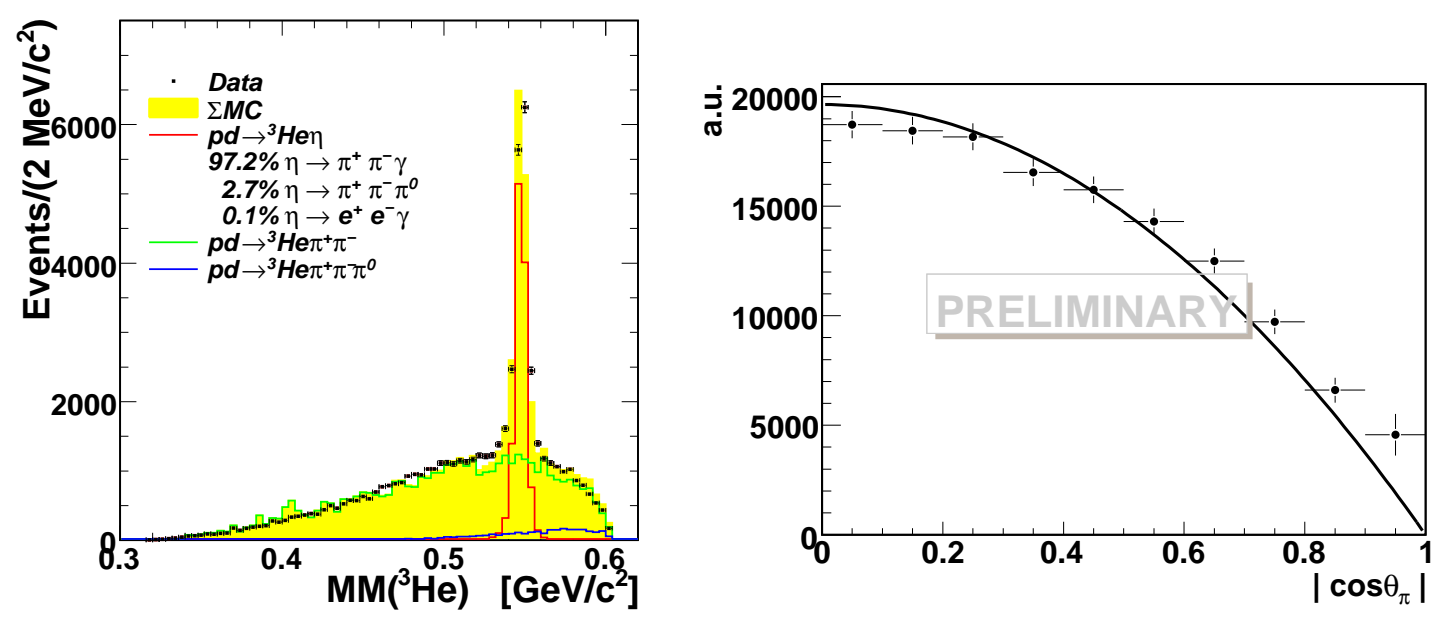

Figure 6: (left) ${ }^{3} \mathrm{He}$ missing mass spectrum for a selection of $\eta \rightarrow \pi^{+} \pi^{-} \gamma$. (right) Preliminary acceptance corrected experimental $d \Gamma / d \cos \theta_{\pi}$ distribution. Line is the expectation from Eqn. (2.3).

mass $\theta_{\pi}$. Since the pions are in $p$ wave one expects the following distribution:

$$
\frac{d \Gamma}{d \cos \theta_{\pi}} \propto \sin \theta_{\pi}^{2}
$$

The distribution is shown in figure 6 together with the experimental points from preliminary analysis of the $p d$ data from 2008. The background due to $p d \rightarrow{ }^{3} \mathrm{He} \pi^{+} \pi^{-}$reaction was subtracted.

From the analysis of the full data set, about $12000 \eta \rightarrow \pi^{+} \pi^{-} \gamma$ events are expected, with an overall reconstruction efficiency of 3-5\%. The photon energy resolution is better than $10 \mathrm{MeV}$.

\subsection{Single and Double Dalitz Decays}

The WASA detector was optimized for the measurement of electron-positron pairs and photons from decays of $\pi^{0}$ and $\eta$ mesons. The performance of the detector can be checked by studying single Dalitz decays $-\eta \rightarrow e^{+} e^{-} \gamma$ and $\pi^{0} \rightarrow e^{+} e^{-} \gamma$. The decays were observed both in $p p$ and $p d$ interactions. Figure 7 shows an example of the $\eta \rightarrow e^{+} e^{-} \gamma$ decay identification in the invariant mass of $e^{+} e^{-} \gamma$ from the $p d$ data. The data from both $p d$ and $p p$ runs should contain about few thousands $\eta \rightarrow e^{+} e^{-} \gamma$ events.

The decay $\eta \rightarrow \pi^{+} \pi^{-} e^{+} e^{-}$, related to $\eta \rightarrow \pi^{+} \pi^{-} \gamma$, allows for more precise tests of the chiral anomaly by comparison with existing ChPT calculations. A high statistics measurement of $\eta \rightarrow$ $\pi^{+} \pi^{-} e^{+} e^{-}$will also allow to provide constrains for a new kind of flavor conserving $\mathrm{CP}$ violation by measuring the asymmetry of the dihedral angle between the pion- and electron planes. The present PDG value for the branching ratio of the $\eta \rightarrow \pi^{+} \pi^{-} e^{+} e^{-}$decay is $4.2 \times 10^{-4}$.

The status of the analysis of the $\eta \rightarrow \pi^{+} \pi^{-} e^{+} e^{-}$decay is shown in Figure 8. A clear signature of the $\eta \rightarrow \pi^{+} \pi^{-} e^{+} e^{-}$decay is seen in the ${ }^{3} \mathrm{He}$ missing mass, corresponding to about 300 events. The line represents polynomial fit to the direct pion production reactions. According to the Monte Carlo simulations, $50 \%$ of the events in the $\eta$ mass peak are from $\eta \rightarrow \pi^{+} \pi^{-} e^{+} e^{-}$.

The major background contributions come from the $\eta \rightarrow \pi^{+} \pi^{-} \gamma$ decay, with $\gamma$ conversion in the beam tube. Additional background stems from $\eta \rightarrow \pi^{+} \pi^{-} \pi^{0}$, due to internal (Dalitz decay 

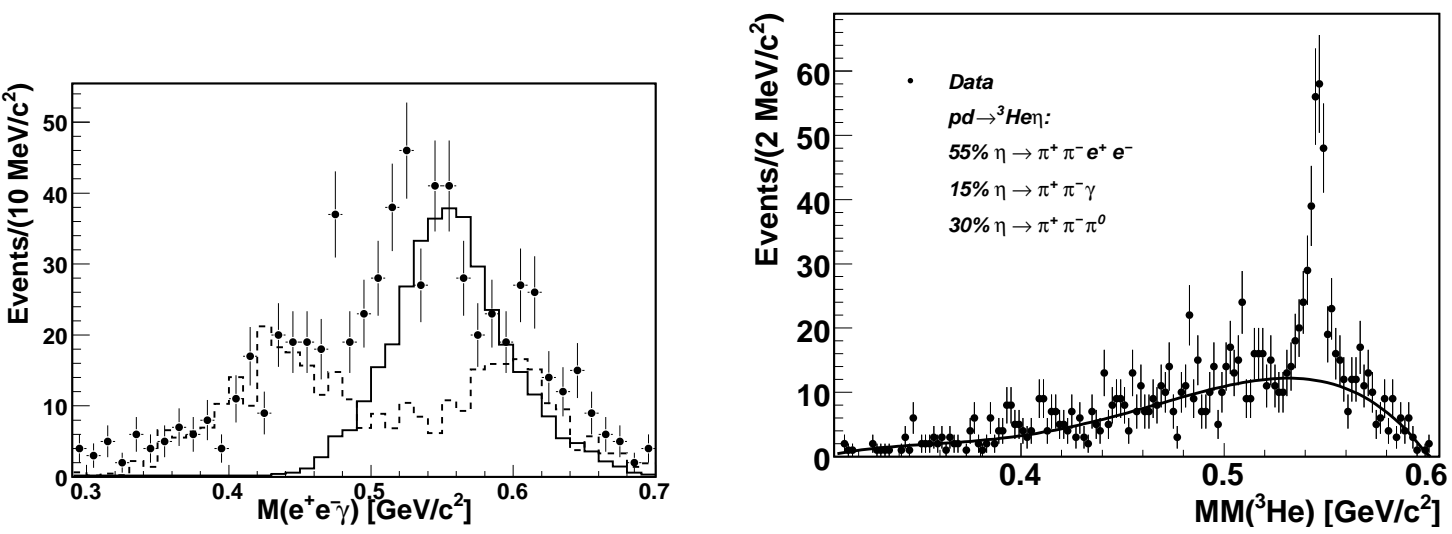

Figure 7: A selection of single Dalitz decay $\eta \rightarrow$ Figure 8: Missing mass of ${ }^{3} \mathrm{He}$ for events compatible $e^{+} e^{-} \gamma$ for about $10 \%$ of the available data. The his- with $\eta \rightarrow \pi^{+} \pi^{-} e^{+} e^{-}$. The line is a polynomial fit to tograms represent Monte Carlo simulation of the sig- the background.

nal (solid line) and background (dashed line).

of the $\pi^{0}$ ) and external conversion of one of the photons from $\pi^{0} \rightarrow \gamma \gamma$ decays. At present, the analysis does not include any vertex reconstruction which will help to suppress the background from external conversion. The remaining background will be dominated by $\eta \rightarrow \pi^{+} \pi^{-} \pi_{\text {Dalitz }}^{0}$ and can be further reduced by improving the detection sensitivity for the additional photon. The overall reconstruction efficiency is limited by requesting the suppression of the background and is 5-10\%.

The measurement of double Dalitz $\eta$ decays, $\eta \rightarrow e^{+} e^{-} e^{+} e^{-}$allows the study of the $\eta$ meson form factor for two timelike virtual photons. In the data collected in 2008 we see signal of about 15 events.

\subsection{Towards rare decays $\eta \rightarrow \pi^{0} e^{+} e^{-}$and $\eta \rightarrow e^{+} e^{-}$}

The $\eta$ decay into $\pi^{0} e^{+} e^{-}$is forbidden to proceed via an intermediate $\pi^{0} \gamma^{\star}$ state due to Cconservation. The present upper limit for the branching ratio is $\leq 4 \times 10^{-5}$. The upper limit would correspond to a maximum of 400 events in the $2008 p d$ run period. No signal is observed in the data. Although the analysis is in an early stage, our sensitivity is already close to the present upper limit. A refined analysis including a full kinematic fit will further suppress the contributions from background channels and enhance the sensitivity.

The dominant mechanism for $\mathscr{P} \rightarrow \ell^{+} \ell^{-}$decays within the Standard Model is a process involving two virtual photons and additionally suppressed by helicity conservation. Recently the interest in the decays was revived due to observed excess for $\pi^{0} \rightarrow e^{+} e^{-}$decay branching ratio [22]. For the $\eta \rightarrow e^{+} e^{-}$decay the best experimental limit $\left(2.7 \times 10^{-5}\right)$ comes from CELSIUS/WASA [5].

\section{References}

[1] H. H. Adam et al. [WASA-at-COSY Collaboration], arXiv:nucl-ex/0411038.

[2] C. Bargholtz et al. [CELSIUS/WASA Collaboration], Nucl. Instrum. Meth. A 594 (2008) 339 [arXiv:0803.2657 [nucl-ex]]. 
[3] C. Adolph et al. [WASA-at-COSY Collaboration], Phys. Lett. B 677 (2009) 24 [arXiv:0811.2763 [nucl-ex]].

[4] C. Bargholtz et al. [CELSIUS-WASA Collaboration], Phys. Lett. B 644 (2007) 299 [arXiv:hep-ex/0609007].

[5] M. Berlowski et al., Phys. Rev. D 77 (2008) 032004.

[6] M. Bashkanov et al., Phys. Rev. C 76 (2007) 048201 [arXiv:0708.2014 [nucl-ex]].

[7] J. Gasser and H. Leutwyler, Nucl. Phys. B 250 (1985) 539.

[8] H. Osborn and D. J. Wallace, Nucl. Phys. B 20 (1970) 23.

[9] J. Bijnens and K. Ghorbani, JHEP 0711 (2007) 030 [arXiv:0709.0230 [hep-ph]].

[10] J. Kambor, C. Wiesendanger and D. Wyler, Nucl. Phys. B 465 (1996) 215 [arXiv:hep-ph/9509374].

[11] N. Beisert and B. Borasoy, Nucl. Phys. A 716 (2003) 186 [arXiv:hep-ph/0301058].

[12] B. Borasoy and R. Nissler, Eur. Phys. J. A 26 (2005) 383 [arXiv:hep-ph/0510384].

[13] W. B. Tippens et al. [Crystal Ball Collaboration], Phys. Rev. Lett. 87 (2001) 192001.

[14] S. Giovannella et al. [KLOE Collaboration], arXiv:hep-ex/0505074.

[15] F. Ambrosino et al. [KLOE collaboration], arXiv:0707.4137 [hep-ex].

[16] M. Unverzagt et al. [Crystal Ball at MAMI Collaboration and TAPS Collaboration and A2 Collaboration], Eur. Phys. J. A 39 (2009) 169 [arXiv:0812.3324 [hep-ex]].

[17] S. Prakhov et al. [Crystal Ball Collaboration at MAMI and A2 Collaboration], Phys. Rev. C 79 (2009) 035204 [arXiv:0812.1999 [hep-ex]].

[18] C. O. Gullstrom, A. Kupsc and A. Rusetsky, Phys. Rev. C 79 (2009) 028201 [arXiv:0812.2371 [hep-ph]].

[19] J. R. Batley et al. [NA48/2 Collaboration], Phys. Lett. B 633 (2006) 173 [arXiv:hep-ex/0511056].

[20] F. Ambrosino et al. [KLOE Collaboration], JHEP 0805 (2008) 006 [arXiv:0801.2642 [hep-ex]].

[21] E. Abouzaid et al. [KTeV Collaboration], Phys. Rev. D 75 (2007) 012004 [arXiv:hep-ex/0610072].

[22] A. E. Dorokhov and M. A. Ivanov, Phys. Rev. D 75 (2007) 114007 [arXiv:0704.3498 [hep-ph]]. 